
3 Research Square
Preprints are preliminary reports that have not undergone peer review.
They should not be considered conclusive, used to inform clinical practice, or referenced by the media as validated information.

\title{
The Molecular Mechanism of Targeting the NF-KB Signaling Pathway to Modulate Mycoplasma Ovipneumoniae Infection in Sheep Based on Transcriptome Sequencing
}

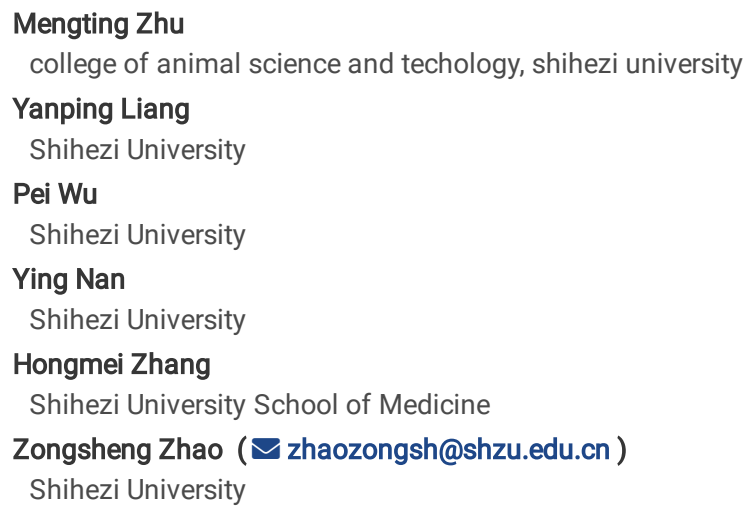




\section{Abstract}

Background: MicroRNAs (miRNAs) play a key role in Mannan-binding lectin (MBL)-mediated resistance to Mycoplasma ovipneumoniae (MO) pneumonia (MP), by regulating the translation of mRNAs of target genes, thereby regulating the immune response. Sheep with different MBL genotypes have different plasma MBL levels, and show varied resistance to MP.

Results: Using previously constructed high-throughput sequencing and transcriptome databases, miRNA-mRNA joint analysis showed that miR-509-5p is differentially expressed. miR-509-5p target genes were predicted using bioinformatic analysis, among which TRAF6 was selected. A TRAF6 dual luciferase reporter vector was co-transfected into $293 \mathrm{~T}$ cells and sheep respiratory mucosal epithelial cells to detect and analyze changes in luciferase activity. qRT-PCR was used to analyze the effect of miR-509-5p on the expression and regulation of TRAF6 and other genes related to the NF-kB signaling pathway. miR-509-5p mimic and miR-509-5p mimic NC were transfected into susceptible sheep respiratory mucosal epithelial cells, for MO challenge infection experiments. The expression levels of cytokines, TRAF6 and other related genes in the NF-KB pathway were analyzed by ELISA and qRT-PCR.

Conclusion: The results proved that miR-509-5p targets TRAF6 to affect the expression of downstream genes and cytokines, which negatively regulates the NF-KB pathway, thereby affecting the inflammatory response.

\section{Background}

Mycoplasma pneumonia (MP), also known as sheep contagious pleuropneumonia, is caused by Mycoplasma ovipneumoniae (MO) infection and can infect sheep at 1-3 months old. MP is a chronic respiratory infection of sheep, characterized by coughing, wheezing, and chronic proliferative interstitial pneumonia. MP affects sheep worldwide and is currently highly prevalent in Xinjiang province, China [1,2]. Surveys of several large-scale intensive sheep farms in Shihezi City and Manas County, Xinjiang, showed that the incidence of the disease had increased because of the introduction of large numbers of Hu sheep and small-tailed sheep. The average morbidity rate is as high as $38 \%$, and the mortality rate in several sheep farms exceeds $20 \%$, especially for lambs. In addition, because of the high variability of the Mycoplasma antigen protein and the unclear pathogenic mechanism and immune evasion mechanism, most immunological and genetic diagnosis techniques have restricted utility [3]. Mycoplasma lacks a cell wall, thus most antibiotics have no significant inhibitory effect, and existing vaccines and therapeutic drugs are not effective [4]. Therefore, MP has a huge impact on intensive sheep farms and is an important bottleneck restricting sheep production in Xinjiang province. Currently, researchers are investigating new ideas and methods to prevent and treat MP. The use of natural immune regulators to develop new vaccines, especially the mannan binding lectin (MBL), is of great significance to improve the body's immune activity and resisting invasion by foreign pathogens.

$\mathrm{MBL}$, as a cytomodulin, can initiate a non-specific immune response, can directly or indirectly bind complement, and is expressed in plasma [5]. Previous studies have found that MBL plays an important role in the protection of humans against Mycoplasma infection [6]. Halbrich [7] found that MBL immunodeficiency in children with mycoplasma pneumonia was significantly higher than that in the control group. Similarly, a research group constructed in vitro $\mathrm{MO}$ and tracheal epithelial cell infection models. By detecting the expression of related immune factors, the authors found that MBL exerts an immune effect before the cells MO and cells were combined [6]. Zhao [3] also confirmed the relationship between MBL protein and MP resistance.

Interestingly, differentially expressed microRNAs (miRNAs) have been suggested to be involved in the molecular regulatory mechanism of disease resistance and might play an anti-disease role by targeting immune signaling pathways. miRNAs are a type of small, endogenous non-coding RNAs that participate in various regulatory pathways, including development, virus defense, cell proliferation, and apoptosis [8,9]. Recent evidence shows that miRNAs play a decisive role in immune regulation and cell development $[10,11]$. Some miRNAs regulate and participate in the innate immune response and inflammatory response, and thus might regulate immune diseases. For example, Zhong and colleagues [12] found that after overexpression of miR-143, cell adhesion was inhibited. Kim [13] found that miR145 could inhibit the growth of breast cancer cells. In addition, in a study of tissue expression profiles of liver cancer, miR-375-5p was observed to inhibit the migration, invasion, epithelial-mesenchyme transition (EMT), and metastasis of liver cancer cells [14]. miR-509-5p is related to the survival rate of pancreatic cancer patients and has been identified as an independently selected mortality predictor. In addition, miR-509-5p can be used as a biomarker for pancreatic cancer. Moreover, miR-509-5p plays an important role in immune regulation, but it has not received much attention [15]. At present, there are no reports on the key genes and key cytokines that regulate MBL-mediated resistance to MP. Furthermore, it is not clear whether this resistance mechanism functions via the nuclear factor kappa B (NF-kB) signaling pathway.

This study was based on a small RNA sequencing library and a transcriptome sequencing library that were constructed in an earlier stage of our research (Unpublished). Using these libraries, target genes were screened using miRNA-mRNA joint analysis. At the same time, we analyzed the expression of related genes in the upstream and downstream pathways of inflammatory response and the changes of various immune factors, which prompted us to speculate that MBL participates in immune regulation pathways. Therefore, we verified the role of regulatory genes in the MBL-mediated anti-infection mechanism. The results of the present study provide a basis for further investigation of the mechanism of resistance to MP disease, and will provide practical advice and assistance for research into MP resistance in certain areas of Xinjiang province.

\section{Results}

miRNA-mRNA joint analysis and prediction of target genes

Six miRNAs were selected randomly using U6 as an internal reference for qRT-PCR verification. The miRNA expression trends were basically the same as Solexa sequencing results, thus confirming the quality of the high-throughput sequencing results (Fig.1A). Through miRNA-mRNA joint analysis, it was determined that miR-509-5p was differentially expressed, and the prediction software identified that TRAF6 may be the target gene.(Fig.1B) 
TargetScan and RNAhybrid2.2 software predicted the target gene of oar-miR-509-5p as TRAF6. We then analyzed binding site of oar-miR-509-5p in the TRAF6 3'-UTR. As shown in Figure 2A, after electrophoresis of the bacterial PCR amplification product, the position of the band was consistent with the expected size of the TRAF6 3'-UTR target fragment. The fragment was cloned into psiCHECK囚-2 and sequenced. A comparison between the sequence of the ligated product and the original sequence showed that they were consistent, indicating that the psiCHECK囚-2-TRAF6 reporter vector was successfully constructed.

Shanghai Biotech produced the mutated version of the TRAF6 3'-UTR lacking the oar-miR-509-5p binding site. This fragment was cloned into psiCHECKX-2 and verified by double enzyme digestion with QuickCut ${ }^{\mathrm{TM}}$ Not I and QuickCut ${ }^{\mathrm{TM}}$ Xho I. Further PCR amplification showed that the fragment was in the correct position in psiCHECK囚-2, indicating that the psiCHECK囚-2-TRAF6-mut reporter vector was successfully constructed (Figure 2B) and could be used in the subsequent experimental research.

According to Fig. $2 \mathrm{C}$ and D, sequencing of the inserts in both vectors, followed by alignment, further confirmed that reporter vectors psiCHECKX-2-TRAF6 containing the oar-miR-509-5p binding site and the psiCHECKX-2-TRAF6-mut lacking the miR-509-5p binding site had been successfully constructed.

\section{$293 T$ cell culture transfection}

In this experiment, 293T cells were selected for transfection. The cells were in a good state before transfection, and the cells displayed their characteristic irregular shape (Fig. 3A). The cells transfected with the various constructs detailed in section 2.3 cells maintained their shape, which was similar to that of the cells before transfection, and their growth status was better (Fig. 3B).

\section{Effect of oar-miR-509-5p on the expression of TRAF6}

The successfully constructed wild-type psiCHECK囚-2-3'-UTR-TRAF6 and mutant psiCHECK囚-2-3'-UTR-TRAF6-M dual luciferase reporter vectors were transfected together with miRNA mimics and negative controls, respectively, into 293T cells. A BioTek microplate reader detected the results of the dual fluorescence reporting system. The mimic negative control, psiCHECKX-2-3'-UTR-TRAF6, and its mutant co-transformation group were used as negative controls. The results (Fig. 4) showed that the cells transfected with wild-type plasmid and the miR-509-5p mimic expressed less luciferase activity compared with that of the control group $(P<0.01)$. However, the cells transfected with the mutant vector expressed similar amounts of luciferase to the control, regardless of whether they were co-transfected the miR-509-5p mimic or its NC. Based on these results, TRAF6 may be the target gene of miR-509-5p.

\section{Morphological observation and immunohistochemical staining of mucosal epithelial cells from the sheep respiratory tract}

According to the research method, primary cell culture was carried out to obtain two kinds of cells: Sheep airway mucosa epithelial cells and fibroblasts. Microscopic examination revealed that the epithelial cells had no fixed shape and are arranged like paving stones, which are easy to gather together, whereas the fibroblasts appear as spindles (Fig. 5A). After $48 \mathrm{~h}$, the purified airway mucosal epithelial cells covered the bottom of the culture dish, as shown in Figure 5B. Immunohistochemistry showed that these cells were positive for keratin 11, identifying them as respiratory mucosal epithelial cells (dark brown cytoplasm and blue nuclei). The results showed that the cells cultured using the tissue block adherence method are the cells required for the experiment (Fig. $5 \mathrm{C}$ ).

\section{Effect of miR-509-5p on the NF-KB pathway related genes}

To further investigate the regulation of miRNA on the 3' UTR region of the target gene TRAF6 and pathway-related genes, qRT-PCR was used to detect their expression in sheep respiratory mucosa cells treated with the miRNA mimic, the mimic NC, the miRNA inhibitor, and inhibitor NC. Compared with that in the miR-509-5p mimic NC transfection group, TRAF6 expression was significantly downregulated in the miR-509-5p mimic transfection group ( $P<0.01)$. The expression levels of other genes also varied, among which TLR4 (toll like receptor 4) and IRAK4 (interleukin 1 receptor associated kinase 4 ) expression levels were significantly upregulated $(\mathrm{P}<0.05)$, TAK1 (TGF-beta-activated kinase 1) expression was significantly downregulated $(\mathrm{P}<0.05), N F K B(N F-K B)$ expression was significantly downregulated $(\mathrm{P}<0.01)$, and TIRAP (TIR domain containing adaptor protein) and TAB2 (TGF-beta activated kinase 1 (MAP3K7) binding protein 2) expression levels were not significantly different (Fig. 6)

\section{SNP analysis of MBL sequencing results}

Cells were isolated from susceptible "BB" type sheep and exon 1 of the $M B P$ gene was sequenced. Analysis of the BB sequence compared with that of the $M B P$ sequence deposited in the NCBI database (accession number fj97762) showed that the 105th base in the "BB" type sequence is T compared with $C$ in the NCBI sequence (Fig. 7).

\section{Detecting the cell death rate by PI staining}

The results of $\mathrm{PI}$ staining to calculate the cell death rate of each group are shown in Table 1. Compared with the control group, the cell death rate of the MO group increased by $32.47 \%(P<0.05)$; while the death rate of the $M O+M B L$ group increased by $6.6 \%(P>0.05)$. The death rate in the $M 0$ group increased by $25.87 \%$ compared with that in the $\mathrm{MO}+\mathrm{MBL}$ group $(\mathrm{P}<0.05)$.

Table 1 Cell Mortality rate of in each group by PI counting 


\begin{tabular}{|ll|}
\hline group & Cell Mortality rate $₫ \% \rrbracket$ \\
\hline Blank control group & $4.23 \pm 0.45^{\mathrm{a}}$ \\
\hline MO group & $36.70 \pm 1.73^{\mathrm{b}}$ \\
\hline MO+MBL group & $10.83 \pm 0.35^{\mathrm{a}}$ \\
\hline
\end{tabular}

Note: Different lowercase letters indicate significant difference $(P<0.05)$, and the same letter indicates that the difference is not significant $(P>0.05)$.

Detection of IL-12, TNF-a, IL-6, IL-1 $\beta$ levels in cell culture fluid

When the cells are infected by MO, they produce an inflammatory response. According to Figure 8A, the levels of IL-12 in the two control groups were significantly different $(\mathrm{P}<0.05)$, indicating that after $\mathrm{MO}$ infection, the expression of cytokine IL-12 increases, suggesting a severe immune reaction. Furthermore, the IL-12 level in blank control group was significantly increased compared with that in the miR-509-5p mimic group ( $<<0.01)$, and was significantly decreased compared with that in the miR-509-5p mimic NC group $(P<0.01)$, indicating that miR-509-5p was overexpressed. The IL-12 level in the experimental control group was significantly increased compared with that in the miR-509-5p mimic group $(P<0.05)$, and the level in the miR-509-5p mimic group was significantly decreased compared with that in the miR-509-5p mimic NC group $(P<0.01)$. The differences in IL-12 levels between miR-509-5p mimic NC groups were not significant. Figure 8B shows that when the cells were infected with MO without any miRNA treatment, the level of TNF- $a$ in the cell culture supernatant was significantly increased. The levels of TNF-a in the blank control group and miR-509-5p mimic group were significantly different (P< 0.05$)$; and the TNF-a levels in the miR-509-5p mimic group and miR-509-5p mimic NC group were significantly different $(P<0.01)$. There was no significant difference among the experimental control group, miR-509-5p mimic NC group, and blank control group. The expression levels of IL-6 in cell culture medium are shown in Figure 8C. The IL-6 levels of the blank control group and the miR-509-5p mimic group were significantly different $(P<0.01)$; the level in the experimental control group was significantly decreased compared with that in the miR-509-5p mimic group $(P<0.01)$. Compared with the IL-6 level in the miR$509-5 p$ mimic NC group, the level in the miR-509-5p mimic group was statistically significantly decreased $(P<0.05)$. The trends of levels of IL-1 $\beta$ in cell the culture medium among the groups were roughly consistent with those of IL- 12 and IL-6. Compared the IL-1 $\beta$ levels between the miR-509-5p mimic group and the miR-509-5p mimic NC group, showed a statistically significant difference $(P<0.05)$, while the IL-1 $\beta$ level in the blank group was not significantly different from that in the other three groups. The IL-1 $\beta$ level in the experimental control group was not significantly different from that in the miR-509-5p mimic group and miR-509-5p mimic NC group. (Fig. 8D)

\section{Effect of MO on mRNA Expression of TRAF6-Related Genes}

As shown in Figure 9, the expression levels of TRAF6 and NF-KB-related genes increased significantly after MO infection, while the expression levels of other genes were also significantly higher than in the blank control group. This showed that $\mathrm{MO}$ infection of respiratory tract mucosal epithelial cells could increase the expression of NF-KB signaling pathway related genes to varying degrees. Compared with that in the miR-509-5p mimic NC group, overexpression of miR509-5p resulted in decreased expression of downstream genes of the pathway. Among them, the expression levels of TRAF6 and NF-KB genes were significantly reduced $(\mathrm{P}<0.01)$, as was the expression level of TAK1 $(\mathrm{P}<0.05)$. Thus, miR-509-5p might target TRAF6 to affect downstream gene expression.

\section{Discussion}

miRNAs function by binding to specific nucleotide sequences in their target mRNAs to exert their gene regulation effect. Each miRNA may have multiple target genes, and an mRNA can be affected by multiple miRNAs [Channakkar et al., 2020; Jeffries et al., 2019; Shuihong et al., 2019][17]. In the present study, the target genes of miRNAs were predicted, and the intersection with the differentially expressed genes was determined, which greatly reduced the search range. Currently, miRNA-mRNA association analysis has opened up new ways of thinking and provided new perspectives to reveal key genes and related signaling pathways of complex traits or complex diseases. Many studies have conducted miRNA-mRNA joint analysis on humans and domestic animals, such as chickens and pigs. Wang and his colleagues used miRNA-mRNA joint analysis and found that growth hormone (GH) regulates the lipid metabolism in chicken liver by regulating the expression of certain mRNAs and miRNAs involved in the regulation of lipid metabolism [18]. Li et al. performed miRNA-mRNA joint analysis on miRNA-mRNA data obtained from the spleens of Yorkshire pigs to obtain miRNAs and genes related to the immune response. The results showed their potential role in the difference in disease resistance between different pig breeds and stages, providing useful information for disease resistance breeding [19]. In humans, the molecular mechanism of male prostate cancer, the second most common cancer, was revealed by combining miRNA-mRNA expression profiles. The identification of key genes and valuable biological processes could be used to understand the molecular mechanism of treatments targeting prostate cancer [20]. Furthermore, using multi-omics data, the molecular mechanism of cervical cancer was explored, and key related genes and biological processes were obtained, which provided an important basis for understanding the molecular mechanism of this cancer [21]. The joint analysis of miRNAmRNA can more accurately screen the key genes that regulate the trait of interest. Currently, joint analysis has become a popular and effective method to study the molecular mechanism of certain traits, however, this technique has not been applied sheep disease resistance breeding traits. Therefore, the present study was based on previously constructed high-throughput sequencing and transcriptome databases. Using miRNA-mRNA joint analysis in these databases, we predicted that TRAF6 might be the target gene of miR-509-5p. Next, we constructed wild-type and mutant TRAF6 3'-UTR region dual luciferase reporter gene vectors to verify the targeting relationship between miR-509-5p and TRAF6. Furthermore, combined with analysis of the expression of related genes in the upstream and downstream pathways of the inflammatory response and the changes of various immune factors, we speculated on the composition of the immunoregulatory pathway of MBL, and verified the role of regulatory genes in the mechanism of MBL-mediated anti-infection.

Tumor necrosis factor receptor-related factor 6 (TRAF6) is a member of the TRAF family and is widely involved in the inflammatory process. In addition, TRAF6 is closely related to the activation of many inflammatory signaling pathways. Without TRAF6, many inflammatory signaling pathways cannot be 
activated [22]. Previous studies have shown that miR-146a directly regulates TRAF6 transcripts by binding to multiple sites in its 3' UTR to regulate immune function. Although miR-146a-dependent degradation of TRAF6 mRNA prevents the production of new TRAF6 protein, existing proteins can continue to function. Studies have shown that TRAF6 can affect the inflammatory response as part of the NF-KB signaling pathway [23,24]. miR-146a directly targets TRAF6 in peripheral blood mononuclear cells (PBMCs) of patients with type 2 diabetes, indicating that miR-146a might affect the inflammatory response by regulating TRAF6 [25]. Additionally, there are studies showing that TNFA (TNF- $a$ ) is the target gene of miRNA-509. Overexpression of miR-509 can increase apoptosis and inhibit TNF-a expression [26]. Furthermore, it has been reported that iron overload could reduce the expression of miR-146a and activate the expression of TRAF6, thereby promoting the expression of NF-KB-dependent inflammatory factors [27] and augmenting the occurrence of inflammation (Fig. 10). In this study, we used the Dual-Luciferase ${ }^{\circledR}$ Reporter Assay System and transformed cells with reporter vectors containing the wild-type TRAF6-3' UTR and a mutated version lacking the miR-509-5p binding site. Cotransfection of the reporter constructs with miR-509-5p mimics and NC controls showed that in the presence of the wild-type UTR and the miR-509-5p mimic, the luciferase activity was significantly lower than that of the NC control group. The luciferase activity in the cells with the mutated UTR reporter vector was similar between the miR-509-5p mimic group and its NC control. This confirmed that oar-miR509-5p binds to 3' UTR of TRAF6 through base complementation, further confirming the direct targeting relationship between TRAF6 and miR-509-5p. To further study the regulatory effect of miR-509-5p on the target gene TRAF6 and pathway-related genes, qRT-PCR was used to detect mRNA expression levels of target gene TRAF6 and its signal transduction pathway related genes. Overexpression of miR-509-5p significantly reduced the mRNA expression level of the target gene TRAF6, whereas miR-509-5p inhibitors significantly increased the mRNA expression level of TRAF6. These results suggested that miR-509-5p might play a role in the inflammatory response by targeting TRAF6; however, the regulatory mechanism needs to be further studied to better understand the mechanism resistance to $\mathrm{MO}$ infection.

$\mathrm{MO}$ is a highly pathogenic microorganism that causes infectious pleura pneumonia in sheep and goats. It is widespread and causes serious economic losses to the sheep industry. MO attaches to and damages ciliary cells, which can lead to dry cough, shortness of breath, wheezing, and progressive weight loss. When these symptoms become irreversible, they can cause death of the infected sheep $[27,28]$. MBL is an acute-phase protein synthesized and secreted by the liver. It belongs to the protein family of collagen lectins (collectins) in the C-type lectin superfamily and is widely found in liver and blood. MBL plays a leading role in the initial stage of anti-infection by activating the complement system and regulating phagocytosis. MBL is an important natural immune defense molecule and is important for improving the body's immune activity and resisting the invasion of foreign pathogens [29,30]. Tabona et al. used transgenic mice as the research object and found that MBL played an important immunity-related role in the initial stage of infection. Therefore, MBL might be one of the most important anti-infective immune molecules in the body's non-specific immunity system. Taking bovine MBL as an example, a concentration of $0.02-0.30$ $\mu \mathrm{g} / \mathrm{mL}$ will inhibit the infection and hemagglutination of the virus. Thus, bovine MBL can neutralize the infectivity of the virus and inhibit its spread in the serum at physiological concentrations[31]. Mutation or abnormality in the promoter region of the MBL gene will cause a decrease in the concentration of MBL$A$ in bovine serum or reduce the function of MBL-A binding bacteria, thereby affecting the innate immune function of cattle. In turn, MBL triggers repeated acute and chronic infections of multiple pathogens of the body, causing damage to various organ systems, which might also cause various autoimmune diseases [32]. Yang et al. studied the changes in immune factors after MO infection of sheep with different MBL genotypes. The results showed that a low MBL concentration is related to Mycoplasma ovipneumoniae infection, and the levels of tumor necrosis factor-a, interferon-y, complement C1 and C3 among the four genotypes were also determined, which showed that the MBL A and B types conferred susceptibility, and the $C$ type conferred resistance [33]. In our previous research, the expression levels of various immune factors in different MBL genotypes infected with MO were detected at the individual level [3]. Highly significantly, with the gradual decrease of inflammation, the level of immune factors might be related to the severity of the disease, which is consistent with the results of the present study. After MO infection of susceptible respiratory mucosal epithelial cells, the levels of IL-12, IL-6, IL-1 $\beta$, and TNF-a increased to varying degrees compared with those in the blank control group. Studies have shown that diethylnitrosamine (DEN) exposure promotes the production of IL6 in Kupffer cells (KCs) in a manner dependent on the Toll-like receptor adaptor protein, MyD88, and its ablation also protects male mice from DEN-induced hepatocarcinogenesis [34]. Yang et al. Found that IL-1 $\beta$ can induce the production of inflammatory mediators in HeLa epithelial cell lines, thereby regulating the MAPK signaling pathway [35]. It has been reported that IL-8 plays a key role in the pathogenesis of upper respiratory tract asthma caused by allergies. In addition, IL-8 is expressed in bronchial epithelial cells. Research also indicated that MBL activation of extracellular regulated kinase (ERK) might be one of the mechanisms leading to increased production of IL-8 [36]. Many studies have shown that the promotion cytokines expression and the release of inflammatory factors is an important part of activating the body's inflammatory response [37]. Overexpression and inhibition of miRNA function showed that miRNA-146a could negatively regulate the production of inflammatory factors IL-8 and RANTES [37]. Rossato [38] identified miR-187 as an IL-10-dependent miRNA, which plays a role in IL-10-mediated inhibition of TNF-a, IL-6, and IL-12 p40 subunit (IL-12p40). When miR-187 expression was silenced, LPS-induced TNF-a, IL-6, and IL-12p40 production increased significantly. The results of these studies are consistent with the findings of the present study. MO infection of respiratory mucosal epithelial cells overexpressing miR-509-5p suppressed the expression of cytokines IL-12, TNF-a, IL-6, and IL-1 $\beta$. Thus, we hypothesized that miR-509$5 \mathrm{p}$ might regulate the disease resistance to Mycoplasma ovipneumoniae by targeting the NF-kB signaling pathway. Based on the above test results, a regulatory pathway diagram was drawn (Figure 11).

However, this study only studied from mRNA level of the genes, and did not analyze or discuss the mechanism at the protein level. In addition, in the study of the molecular mechanism of different resistance genotypes of MBL-mediated MO resistance, hepatocytes secreting the MBL protein were not selected for verification experiments; however, the regulatory mechanism in respiratory tract mucosal epithelial cells of the susceptible type (MBL) was discussed. The main reason for choosing these cells is that the respiratory tract mucosal epithelial cells are the host cells infected by Mycoplasma ovipneumoniae. It is not clear whether $\mathrm{MBL}$ regulates the molecular mechanism of disease resistance through miR-509-5p. MBL is an innate immunomodulator and the immune regulation mechanism is extremely complex; therefore, further experimental research is needed to determine the molecular regulatory mechanism of MBLmediated disease resistance.

\section{Conclusions}


The results of our study revealed that there is a true targeting relationship between TRAF6 and miR-509-5p. In addition, when MO infected susceptible respiratory mucosal epithelial cells, the expression levels of genes and related cytokines in the NF-KB signaling pathway increased to varying degrees. Furthermore, overexpression of miR-509-5p resulted the suppression of the expression of downstream genes of the TRAF6 and NF-kB signaling pathways and reduced cytokine IL-12 levels. This suggested that miR-509-5p targeting of TRAF6 affects the expression of downstream genes, thereby achieving disease resistance by targeting the NF-KB signaling pathway to regulate Mycoplasma ovipneumoniae infection, and provides a basis for further study of the molecular regulation mechanism of MBL-mediated disease resistance.

\section{Methods}

\section{Samlpes and cells}

This study was approved by the Ethics Committee of Animal Experiments from the Animal Science and Technology College at Shihezi University. All samples were collected in strict accordance with the committee's guidelines. Ninety-day sheep fetus tracheas were obtained from a live animal slaughtering center in Shihezi, Xinjiang province. The specimens were collected by trained personnel and specialized veterinarians following the animal welfare protocols of the First Affiliated Hospital of the Medical College of Shihezi University and College of animal science and technology. During the experiment, every effort was made to minimize animal suffering.

miRNA-mRNA joint analysis and prediction of target genes

This analysis was based on a high-throughput sequencing database and transcriptome sequencing database established earlier in this project (Unpublished). First, according to the fold-change, P-value, and Q-Value of the high-throughput sequencing database, it was determined that mir-509-5p was differentially expressed. miRNA-mRNA joint analysis also determined that mir-509-5p was a differentially expressed miRNA. TargetScan and RNAhybrid (USA) were used to predict the target genes of the differentially expressed oar-miR-509-5p (Ovis aries miR-509-5p), and the intersection of the results of the two analyses was used as the candidate target genes. Then, combined with sequencing data, Kyoto Encyclopedia of Genes and Genomes (KEGG) pathway analysis, and reference literature for target gene screening, the candidate target genes were identified for subsequent experimental studies.

Identification and construction of target sequence wild-type and mutant psiCHECK ${ }^{T M}-2$ vector

Construction of target sequence wild-type psiCHECK ${ }^{\mathrm{TM}}-2$ vector

The 3'-untranslated region (UTR) of one of the candidate target genes, TRAF6 (encoding TNF receptor associated factor 6 ) was predicted and used to design primers to amplify the wild-type sequence. The primers were designed using Primer 6.0, and added sites for the Not I and Xho I restriction endonucleases to the ends of the amplicon. The $5 \rrbracket$ end bases were protected and the primers were synthesized by Xinjiang Kuntai Rui Company (Table 2). The wild-type 3'-UTR sequence was then cloned into vector psiCHECKX-2 (Promega, USA).

Table 2 Primers information of target gene

\begin{tabular}{|c|c|c|c|}
\hline Gene & Sequence $\left(5^{\prime}-3^{\prime}\right)$ & Size (bp) & $\mathrm{Tm} \rrbracket^{\circ} \mathrm{C} \rrbracket$ \\
\hline \multirow[t]{2}{*}{ TRAF6 } & F:ccgCTCGAGGACTTGCCTTTCACTTTTCA & 490 & 56 \\
\hline & R: tttGCGGCCGCCCTTCAGGTTTCTTTCAC & & \\
\hline
\end{tabular}

Construction of mutant psiCHECK ${ }^{\mathrm{TM}}-2$ vector

Next, the miRNA binding site in the TRAF6 3'-UTR sequence was mutated. The following sequence was designed and cloned into the psiCHECKX-2 vector, which was completed by Shanghai Biotech (China):

\section{CTCGAGCTTTCACTTTTCACTTTCATGCATTGGAGAAGGAAATGGCAACCCACTCCAGTGTTCTTGGCTGGAGAATCCCAGGGACGGGGGAGCCTGGTGGGCTGCCATC}

The sequence in bold is the mutated miRNA binding site in the TRAF6 3'-UTR sequence.

Double Digestion of psiCHECK ${ }^{\mathrm{TM}}-2$ Vector

The psiCHECK\-2 vector contains two reporter genes, the firefly luciferase gene (hluc+) and the Renilla luciferase gene (hluc). In practical use, the Renilla luciferase value is used as an internal reference, and the firefly luciferase activity is used as the reporter.

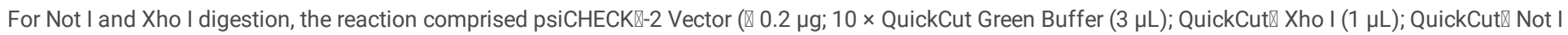
$(1 \mu \mathrm{L})$; and $\mathrm{ddH}_{2} \mathrm{O}$ up to $10-30 \mu \mathrm{L}$. The reaction incubated at $37^{\circ} \mathrm{C}$ for $1 \mathrm{~h}$. The target band was separated by $1.5 \%$ agarose gel electrophoresis, and the product was purified and recovered. The two recovered fragments (wild-type and mutated) were ligated separately into psiCHECKX-2 using the following

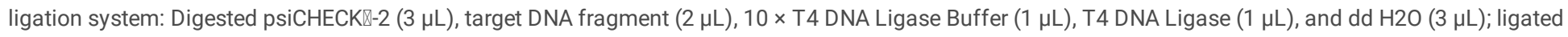
overnight at $4{ }^{\circ} \mathrm{C}$.

$293 T$ cell resuscitation, passage, and transfection

The human embryonic kidney cell line 293T was donated by the College of Animal Science and Technology of Shihezi University. The frozen cells were thawed in a $37^{\circ} \mathrm{C}$ water bath. Then, after sterilizing with $75 \%$ ethanol, the supernatant liquid was discarded, and the appropriate complete medium (Dulbecco's 
modified Eagle's medium (DMEM): Serum: double antibody=90\%:10\%:1\%) was added to the cells in the cryovial tube. The cells were cultured at $37{ }^{\circ} \mathrm{C}$ in $5 \%$ $\mathrm{CO}_{2}$. When the cells reached $70-80 \%$ density, the cell culture fluid is discarded and the cells were digested with a $0.25 \%$ pancreatin solution (Takara, Dalian, China) for 30-60 s. The state of the cells was observed under a microscope. When most of the cells had shed and dispersed, complete culture solution was added to stop digestion. The cells were transferred to a $15 \mathrm{~mL}$ centrifuge tube and centrifuged $g$. The supernatant was discarded and the cells were resuspended in complete medium. Finally, the cells were added to a 6-well culture dish.

To obtain an effective transfection efficiency, the optimal ratio of transfection was determined as 1:2 via pre-experimental plasmid transfection. As observed under an inverted fluorescence microscope, transfection was performed when the cell density reached $70-80 \%$. Then, according to the miRNA product manual (Ruibo Biotechnology Co., Ltd., China), miRNA mimics and inhibitors were formulated as a $20 \mu \mathrm{M}$ stock solution for transfection experiments. The constructs were transfected into five groups of cells: 1 . Control group; 2 . Wild-type psiCHECK\-2 recombinant plasmid + miRNA mimic; 3 . Wild-type psiCHECK囚-2 recombinant plasmid + miRNA Negative Control (NC); 4. mutant psiCHECK囚-2 recombinant plasmid + miRNA mimic; 5. mutant psiCHECK囚-2 recombinant plasmid + NC miRNA. Three parallel wells were set for each group and transfection was performed according to the instructions of the Lipo2000 reagent.

\section{Luciferase activity assay}

The Dual-Luciferase ${ }^{\circledR}$ Reporter Assay System kit (Tiangen, China) was used to assess luciferase activity. At $24 \mathrm{~h}$ after transfection, the culture solution was discarded and the cells were rinsed two to three times with pre-warmed $1 \times$ phosphate-buffered saline (PBS). Then, cell lysis solution (Takara) was added to fully lyse the cells. The lysed cells were transferred to a clean Eppendorf tube, centrifuged, and $20 \mu \mathrm{L}$ of the cell lysate supernatant was added to a Lockwell maxisorp detection plate, followed by $100 \mu \mathrm{L}$ of Luciferase Reaction Reagent. After shaking and mixing, the plate was placed immediately into the chemiluminescence instrument (Zeiss, Oberkochen, Germany) to detect the firefly luciferase activity value. After detecting the firefly luciferase activity value, $100 \mu \mathrm{L}$ of Luciferase Reaction Reagent II was added, and after shaking and mixing, the plate was placed into the chemiluminescence instrument to measure the activity of Renilla luciferase.

\section{Primary culture and purification of sheep respiratory epithelial cells}

Ninety-day-old fetuses were obtained from beef and sheep abattoirs in Shihezi City (Xinjiang Uygur Autonomous Region, China) and their tracheas were collected rapidly. The tracheas were rinsed with PBS buffer two to three times, cut into small pieces and spread on the bottom of a Petri dish. Subsequently, culture medium (DMEM: Serum: double antibody=90\%:10\%:1\%) was added and the plates were placed at $37{ }^{\circ} \mathrm{C}$ in $5 \% \mathrm{CO}_{2}$ for culture; the medium was changed every $48 \mathrm{~h}$. After 3-4 days of culture, the mucosal epithelial cells of sheep respiratory tract were purified. First, the tissue block was removed from the culture medium and rinsed with PBS two to three times. The PBS was discarded and $2 \mathrm{~mL}$ of trypsin $(0.25 \%)$ without EDTA was added to each petri dish, and the tissue was digested at $37^{\circ} \mathrm{C}, 5 \% \mathrm{CO}_{2}$ for $2 \mathrm{~min}$. Culture solution was added to stop the reaction. At this point, most of the fibroblasts had adhered to the wall, while the respiratory mucosal epithelial cells did not adhere. By repeating this method multiple times, purified airway mucosal epithelial cells could be obtained.

\section{Cellular immunohistochemistry}

The cell slides were fixed in $4 \%$ paraformaldehyde solution, dried, incubated with $3 \% \mathrm{H}_{2} \mathrm{O}_{2}$ at room temperature for 10 min, and washed three times with PBS. The slides were then incubated with $0.3 \%$ Triton X-100 for 20 min, and then with PBS-diluted goat serum blocking solution for 40 min. Primary antibodies recognizing keratin 11 (Anti-pan Cytokeratin antibody [C-11], Abcam, Cambridge, MA, USA) were added, and the slides were incubated at $37^{\circ} \mathrm{C}$ for $2 \mathrm{~h}$, and washed three times with PBS. Then, an equal amount of horseradish peroxidase-labeled secondary antibody was added, incubated at $37^{\circ} \mathrm{C}$ for 60 min, and washed three times with PBS. Finally, the cells on the slides were stained using a 3,3'-Diaminobenzidine (DAB) staining kit. The cells were then counterstained with hematoxylin for $30 \mathrm{~s}$ to $1 \mathrm{~min}$ at room temperature for $1 \mathrm{~min}$, and washed with PBS for $10 \mathrm{~s}$. The slides were sealed with gum and examined under a microscope.

\section{Measuring target genes and pathway genes mRNA expression}

Total RNA was extracted from cells and isolated using the TRIzol reagent (Invitrogen). One microgram of total RNA was used to perform reverse transcription using a PrimeScript RT Reagent Kit with gDNA Eraser (Takara) according to the manufacturer's instructions. The primers for all target genes are shown in Table 3. Quantitative real-time polymerase chain reaction (qPCR) was performed using a LightCycler 96 Real-Time qPCR System (Roche, CH). The $20 \mu \mathrm{L}$ amplification reaction mixture included $1.0 \mu \mathrm{L}$ of cDNA, $10 \mu \mathrm{L}$ of $2 \times$ SYBR Premix Ex Taq, $1 \mu \mathrm{L}$ of primers $(0.5 \mu \mathrm{L}$ of each forward and reverse primer), and 8 $\mu \mathrm{L}$ of water. The reactions were performed at $94^{\circ} \mathrm{C}$ for 5 minutes; followed by 40 cycles at $94^{\circ} \mathrm{C}$ for 20 seconds and at $60{ }^{\circ} \mathrm{C}$ for 34 seconds. All reactions were performed in triplicate, and the relative quantification of mRNA was performed using the $2^{-\triangle \Delta \mathrm{Ct}}$ method [16]. The Ct values were used to calculate $\Delta \mathrm{Ct}$ values for genes of interest [Ct (test)-Ct(reference)]. All experiments were performed at least in triplicate.

Table 3 Real -time quantitative PCR primers 


\begin{tabular}{|lllll|}
\hline Gene & GenelD & Size/bp & Sequence $\left(5^{\prime}\right.$-3 $\left.{ }^{\prime}\right)$ & Tm/ ${ }^{\circ} \mathrm{C}$ \\
\hline TLR4 & FJ977629.1 & 80 & F:TGTGAAGGACATGCCAGTGCTTG & 60 \\
& & & R:TGACAACCGACACGCTGATGATC & \\
\hline TIRAP & NM_001009806 & 101 & F:CCTCAGCAGAGCCGCCTACC & 62 \\
& & & R: GCATGACAGCGTCCTTGACTTGG & \\
IRAK4 & M96845 & 84 & F:CTCAAGTGATGGCGATGACCTCTG & 59 \\
& & & R: CCATCCAAGCAAGCCAGTCTGTC & \\
TRAF6 & X52640 & 82 & F: ACTGAGGCATCTTGAGGAGCATC & 63 \\
& & & R: TTCTGGAAGAGACGCTGGCATTG & \\
\hline TAB2 & X56756 & 114 & F: GGAAGCAGGACTCTAACGCACAG & 58 \\
& & & R:GCCTTGAGGAACTTGAGCTGGTG & \\
\hline TAK1 & FJ958365.1 & 125 & F: TCCGCCGCTTCTTCCTCCTC & 59 \\
& & & R: GCTCCTCTTCCAACAACCTCTTCC & \\
\hline NF-KB & AF038130.1 & 104 & F:ACAAGCCTGTCACAGCCAACATG & 62 \\
& & & R: TGATGGTGAAGGCTCAGGAGGTG & \\
\hline B-actin & U39357 & 108 & F:AGAGCAAGAGAGGCATCC & 60 \\
& & & R:TCGTTGTAGAAGGTGTGGT & \\
\hline
\end{tabular}

F: forward primer; R: reverse primer; Primers were designed according to the sequence of the target genes obtained from GenBank.

Detecting cell mortality using propidium iodide (PI) staining method

Tracheas were collected from on "BB type" sheep (susceptible sheep) identified by our research group (unpublished), and the respiratory mucosal epithelial cells were isolated as detailed in section 2.5 and cultured. When the cell density reached $90 \%$, an infection experiment was conducted. The experiment was divided into three groups: control group, $\mathrm{MO}$ group, and $\mathrm{MO}+\mathrm{MBL}$ group. Among them, recombinant MBL protein was provided by our laboratory; and Mycoplasma ovipneumoniae (MO) was provided by the Animal Medicine Experimental Center, College of Animal Science and Technology, Shihezi University. The measured bacterial count was colony forming units $(\mathrm{CFU})=10^{6}$. The instructions of the PI staining test kit were followed to complete the staining process. In the dark, $20 \mu \mathrm{L}$ of the cell-dye mixture was dropped onto the loading chip, and the chip was inserted into the Adam-mc counter for detection and calculation of cell mortality.

Measurement of IL-12, TNF- $a, I L-6, I L-1 \beta$ release

$\mathrm{MO}$ infection was performed after 24 hours of cell culture. The cell culture medium was collected from the blank control group, the experiment control group (blank control group + MO), the miR-509-5p mimic group (miR-509-5p mimic + MO), and the miR-509-5p mimic NC group (miR-509-5p mimic NC + MO) respectively. According to the manufacturer's instructions, interleukin (IL)-12, tumor necrosis factor alpha (TNF-a), IL-6, and IL-1 $\beta$ cytokine secretion levels were measured using an enzyme-linked immunosorbent assay (ELISA) kit (BIM).

Statistical analysis

Each experiment was performed 3 times. The data were expressed as the mean \pm the standard error and all statistical analysis were performed with SPSS software version 19.0 (IBM Corp., Armonk, NY, USA). Differences between means were accepted as statistically significant at P< 0.05 and as a trend at $0.05<$ $P<0.10$.

\section{Abbreviations}

MO: Mycoplasma ovipneumoniae; MBL: mannose-binding lectin; MP: mycoplasma pneumoniae, miRNAs: micro RNAs; NF-kB: the nuclear factor kappa B; UTR: the 3'-untranslated region; TRAF6: tumor necrosis factor receptor-related factor 6; TNF-a: tumor necrosis factor alpha; TLR4: toll like receptor 4; IRAK4: interleukin 1 receptor associated kinase 4; PCR: polymerase chain reaction; qPCR: real-time fluorescent quantitative PCR

\section{Declarations}

\section{Ethics approval and consent to participate}

This study was approved by First Affiliated Hospital of the Medical College of Shihezi University and college of animal science and technology. All procedures were performed in accordance with the guidelines for the care and use of animals of Shihezi University and with current Chinese legislation.

\section{Consent for publication}


Not applicable.

\section{Availability of data and materials}

The data used and/or analyzed in the current study are available from the corresponding author on reasonable request.

\section{Competing Interests}

We certify that there is no competing of interest with any financial organization regarding the material discussed in the manuscript.

\section{Funding}

This research was supported by grants from the National Natural Science Foundation of China (No: 31460587). All authors participated in writing the final draft of the manuscript and agreed on the final format.

\section{Authors' Contributions}

$\mathrm{MZ}, \mathrm{HZ}$ and $\mathrm{ZZ}$ designed the study. $\mathrm{MZ}$ and $\mathrm{YL}$ performed the animal inoculation trial. $\mathrm{MZ}$ performed the laboratory procedures and bioinformatics analysis. $\mathrm{MZ}, \mathrm{PW}$, and $\mathrm{YN}$ analyzed and interpreted the data. MZ drafted the manuscript. MZ, $\mathrm{HZ}$ and ZZ critically revised the manuscript. All author(s) have read and approved the manuscript.

\section{Acknowledgements}

We are grateful to all the members of the laboratory for their help in sampling and performing the experiments.

\section{Author details}

${ }^{1}$ College of Animal Science and Technology, Shihezi University, Shihezi, Xinjiang 832003, China; ${ }^{2}$ First Affiliated Hospital, School of Medical College, Shihezi University, Shihezi, Xinjiang 832008, China

\section{References}

1. Thomas; E.; Besser; E.; Frances; Cassirer; Kathleen; A.; Potter; Kevin. Epizootic Pneumonia of Bighorn Sheep following Experimental Exposure to Mycoplasma ovipneumoniae. Plos One 2014, 9, 1-9, doi:10.1371/journal.pone.0110039

2. Raghavan, B.; Bavananthasivam, J.; Kugadas, A.; Haldorson, G.J.; Srikumaran, S. Effect of vaccination against pneumonia on the survival of bighorn sheep ( Ovis canadensis ) commingled with carrier animals. Veterinary Microbiology 2017, 203, 56-61, doi:10.1016/j.vetmic.2017.02.013

3. Zhao, F.; Zhao, Z.; Yan, G.; Wang, D.; Ban, Q.; Yu, P.; Zhang, W.; Luo, Y. Polymorphisms in mannose-binding lectin (MBL) gene and their association with MBL protein levels in serum in the Hu sheep. Vet Immunol Immunopatho/ 2011, 140, 297-302, doi:10.1016/j.vetimm.2010.12.009

4. Chopra, S.; Kakar, A.; Gogia, A. Mycoplasma pneumoniae : A forgotten bug. Current Medicine Research and Practice 2020, 10, 82-83, doi:10.1016/j.cmrp.2020.03.009

5. Kalia, N.; Singh, J.; Rauniyar, A.K.; Kaur, M. A meta-analysis of mannose-binding lectin gene polymorphisms with the risk of recurrent vulvovaginal infections. Sci Rep 2020, 10, 6079, doi:10.1038/s41598-020-63261-8

6. Eeva; Sajanti; Kirsi; Gr?ndahl-Yli-Hannuksela; Tommi; Kauko; Qiushui; He; Jukka. Lyme Borreliosis and Deficient Mannose-Binding Lectin Pathway of Complement. Journal of Immunology 2015, 194, 358-363, doi:10.4049/jimmunol.1402128

7. Valdimarsson, H. Infusion of plasma-derived mannan-binding lectin (MBL) into MBL-deficient humans. Biochemical Society Transactions 2003, 31, 768769, doi:Doi 10.1042/Bst0310768, doi:10.1042/BST0310768

8. K.; W.; Witwer. Circulating MicroRNA Biomarker Studies: Pitfalls and Potential Solutions. Clinical Chemistry 2014, 61, 56-63, doi囚 10.1373/clinchem.2014.221341

9. Cao, X.; Wang, Z.; Bi, L.; Bi, C.; Du, Q. Gold nanocage-based surface-enhanced Raman scattering probes for long-term monitoring of intracellular microRNA during bone marrow stem cell differentiation. Nanoscale 2020, 12.

10. Mallick, A.; Ranawade, A.; Gupta, B.P. Role of PRY-1/Axin in heterochronic miRNA-mediated seam cell development. BMC Developmental Biology 2019, 19, 17, doi:10.1186/s12861-019-0197-5

11. Puneet; Seth; Paishiun; Hsieh; Suhib; Jamal; Liwen; Wang; Steven. Regulation of MicroRNA Machinery and Development by Interspecies S-Nitrosylation. Cell 2019, 176, 1014-1025(e12), doi:10.1016/j.cell.2019.01.037

12. Zhong, W.; He, B.F.; Quan, T.Y.; Zhu, C.Q.; Chen, Y.L. Expression of miR-143 in nasopharyngeal carcinoma cell lines and its effect on cell adhesion ability. Journal of Southern Medical University 2013, 33, 582-585.

13. Kim, S.J.; Oh, J.S.; Shin, J.Y.; Lee, K.D.; Sung, K.W.; Nam, S.J.; Chun, K.H. Development of microRNA-145 for therapeutic application in breast cancer. Journal of Controlled Release 2011, 155, 427-434, doi:10.1016/j.jconrel.2011.06.026

14. Chen, J.S.; Huang, J.Q.; Dong, S.H.; Huang, X.H. Effects of microRNA-379-5p on proliferation, migration and invasion of hepatocellular carcinoma cell line. Zhonghua yi xue za zhi 2016, 96, 1450-1453, doi:10.3760/cma.j.issn.0376-2491.2016.18.013

15. Hiramoto, H.; Muramatsu, T.; Ichikawa, D.; Tanimoto, K.; Yasukawa, S.; Otsuji, E.; Inazawa, J. miR-509-5p and miR-1243 increase the sensitivity to gemcitabine by inhibiting epithelial-mesenchymal transition in pancreatic cancer. Sci Rep-Uk 2017, 7, 4002, doi:10.1038/s41598-017-04191-w 
16. Livak KJ, Schmittgen TD. Analysis of relative gene expression data using real-time quantitative PCR and the 2(-Delta Delta C(T)). Method 2001, 25, 402408

17. Channakkar, A.S.; Singh, T.; Pattnaik, B.; Gupta, K.; Adlakha, Y.K. MiRNA-137-mediated modulation of mitochondrial dynamics regulates human neural stem cell fate. Stem Cells 2020, 38:683-697, doi:10.1002/stem.3155

18. Wang, X.; Yang, L.; Wang, H.; Shao, F.; Yu, J.F.; Jiang, H.; Han, Y.; Gong, D.; Gu, Z. Growth Hormone-Regulated mRNAs and miRNAs in Chicken Hepatocytes. Plos One 2014, 9, e112896, doi:10.1371/journal.pone.0112896

19. Xinjian; Li; Ruimin; Qiao; Jianwei; Ye; Mingyu; Wang; Chen; Zhang. Integrated miRNA and mRNA transcriptomes of spleen profiles between Yorkshire and Queshan black pigs. Gene 2018,688, 204-214, doi:10.1016/j.gene.2018.11.077

20. Xing, Z.; Sun, Y.Y.; Peng, W.; Yang, C.; Li, S. Exploration of the molecular mechanism of prostate cancer based on mRNA and miRNA expression profiles. Oncotargets \& Therapy 2017, Volume 10,3225-3232, doi:10.2147/OTT.S135764

21. Zhao, L.; Zhang, Z.; Lou, H.; Liang, J.; Ou, R. Exploration of the molecular mechanisms of cervical cancer based on mRNA expression profiles and predicted microRNA interactions. Oncology Letters 2018, 15, 8965-8972, doi:10.3892/ol.2018.8494

22. Zhong, L.; Cao, F.; You, Q. Effect of TRAF6 on the biological behavior of human lung adenocarcinoma cell. Tumor Biology 2013, 34, 231-239, doi:10.1007/s13277-012-0543-8

23. HäCker, H.; Redecke, V.; Blagoev, B.; Kratchmarova, I.; Hsu, L.C.; Wang, G.G.; Kamps, M.P.; Raz, E.; Wagner, H.; H?Cker, G. Specificity in Toll-like receptor signalling through distinct effector functions of TRAF3 and TRAF6. Nature 2005, 439, 204-207, doi:10.1038/nature04369

24. Dickson, K.M.; Bhakar, A.L.; Barker, P.A. TRAF6-dependent NF-kB transcriptional activity during mouse development. Developmental Dynamics 2010, 231, 122-127, doi:10.1002/dvdy.20110

25. Lim, R.; Barker, G.; Lappas, M. The TLR2 Ligand FSL-1 and the TLR5 Ligand Flagellin Mediate Pro-Inflammatory and Pro-Labour Response via MyD88/TRAF6/NF-KB-Dependent Signalling. American Journal of Reproductive Immunology 2014, 71, 401-417, doi:10.1111/aji.12229

26. Zhang, G.; Liu, Z.; Han, Y.; Wang, X.; Yang, Z. Overexpression of miR-509 Increases Apoptosis and Inhibits Invasion via Suppression of Tumor Necrosis Factor-a in Triple-Negative Breast Cancer Hs578T Cells. Oncology Research 2016, 24, 233, doi:10.3727/096504016X14648701447977

27. Garwood, T.J.; Chadwick; Lehman, P.; Daniel; Jenks, J.A. Removal of chronic Mycoplasma ovipneumoniae carrier ewes eliminates pneumonia in a bighorn sheep population. Ecology and Evolution 2020, 10, 1-12, doi:10.1002/ece3.6146

28. Manlove, K.; Branan, M.; Baker, K.; Bradway, D.; Cassirer, E.F.; Marshall, K.L.; Miller, R.S.; Sweeney, S.; Cross, P.C.; Besser, T.E. Risk factors and productivity losses associated with mycoplasma ovipneumoniae infection in united states domestic sheep operations. Preventive veterinary medicine 2019, 168:3038, doi:10.1016/j.prevetmed.2019.04.006

29. Zhou, J.; Hu, M.; Li, J.; Liu, Y.; Chen, Z. Mannan-Binding Lectin Regulates Inflammatory Cytokine Production, Proliferation, and Cytotoxicity of Human Peripheral Natural Killer Cells. Mediators of Inflammation 2019, 2019, 6738286, doi:10.1155/2019/6738286

30. Li, H.; Liu, Y.; Li, J.; Liu, Y.; Dong, L.; Yin, Y.; Yu, Y.; Zhou, J.; Zhang, L.; Lu, X. Mannan-binding lectin attenuates acetaminophen-induced hepatotoxicity by regulating CYP2E1 expression via ROS-dependent JNK/SP1 pathway. European Journal of Immunology 2019, doi:10.1002/eji.201847830

31. Wang X, Ju Z , Huang $J$, et al. The relationship between the variants of the bovine MBL2 gene and milk production traits, mastitis, serum MBL-C levels and complement activity. Veterinary Immunology \& Immunopathology, 2012, 148(3-4), doi:10.1016/j.vetimm.2012.06.017

32. Garzon, R.; Fabbri, M.; Cimmino, A.; Calin, G.A.; Croce, C.M. MicroRNA expression and function in cancer. Trends in Molecular Medicine 2006, 12, 580-587, doi:10.1016/j.molmed.2006.10.006

33. Zhang, H.; Zhao, Z.S.; Zhao, F.; Peng, Y.U. The relationship between MBL genotype and lung histopathologic lesions of Mycoplasma ovipneumoniae pneumonia in sheep. Chinese Journal of Preventive Veterinary Medicine 2012, 34:293-296.

34. Naugler, W.E.; Sakurai, T.; Kim, S.; Maeda, S.; Kim, K.; Elsharkawy, A.M.; Karin, M. Gender disparity in liver cancer due to sex differences in MyD88dependent IL-6 production. Science 2007, 317, 121-124.

35. Yang, H.T.; Cohen, P.; Rousseau, S. IL-1 $\beta$-stimulated activation of ERK1/2 and p38a MAPK mediates the transcriptional up-regulation of IL-6, IL-8 and GRO-a in HeLa cells. Cellular Signalling 2008, 20,375-380, doi:10.1016/j.cellsig.2007.10.025

36. Sohn, M.H.; Lee, K.E.; Choi, S.Y.; Kwon, B.C.; Chang, M.W.; Kim, K.E. Effect of Mycoplasma pneumoniae lysate on interleukin-8 gene expression in human respiratory epithelial cells. Chest 2005, 128, 322-326, doi:10.1016/S0012-3692(15)37964-2

37. Na; Li; Xiang; Xu; Bin; Xiao; En-Dong; Zhu; Bo-sheng; Li. H. pylori related proinflammatory cytokines contribute to the induction of miR-146a in human gastric epithelial cells. Molecular Biology Reports 2012, 39:4655-4661, doi:10.1007/s11033-011-1257-5

38. Marzia, R.; Graziella, C.; Nicola, T.; Monica, C.; Laura, M.; Sara, G.; Barbara, M.; Mariacristina, D.L.; Massimiliano, M.; Marco A, C. IL-10-induced microRNA187 negatively regulates TNF-a, IL-6, and IL-12p40 production in TLR4-stimulated monocytes. Proc Natl Acad Sci U S A 2012, 109, 18257-18258, doi:10.1073/pnas.1209100109

\section{Figures}


A

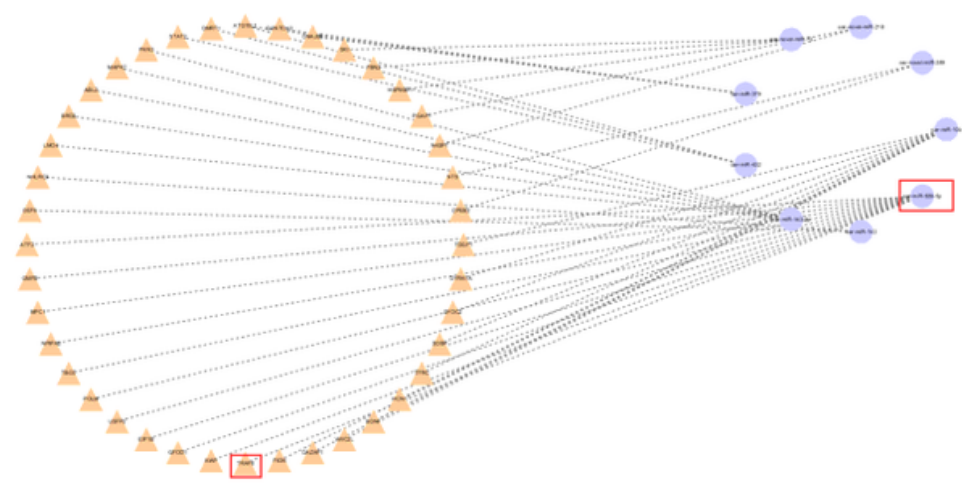

B

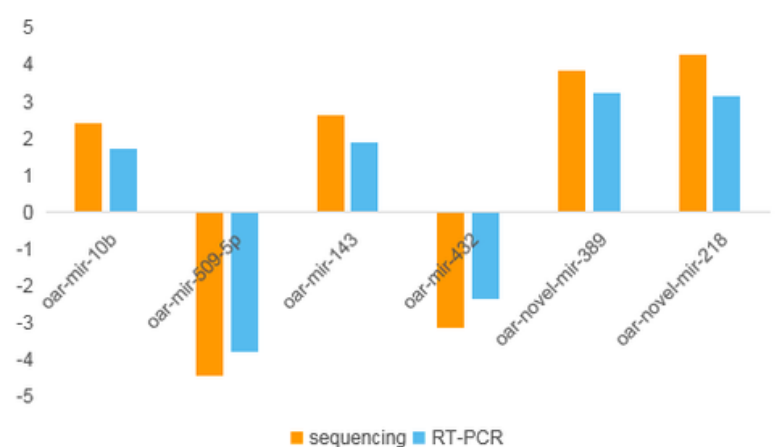

Figure 1

miRNA-mRNA joint analysis and target gene prediction. (A) miRNA-mRNA joint analysis. (B) Validattion of the deep sequencing results using qRT-PCR
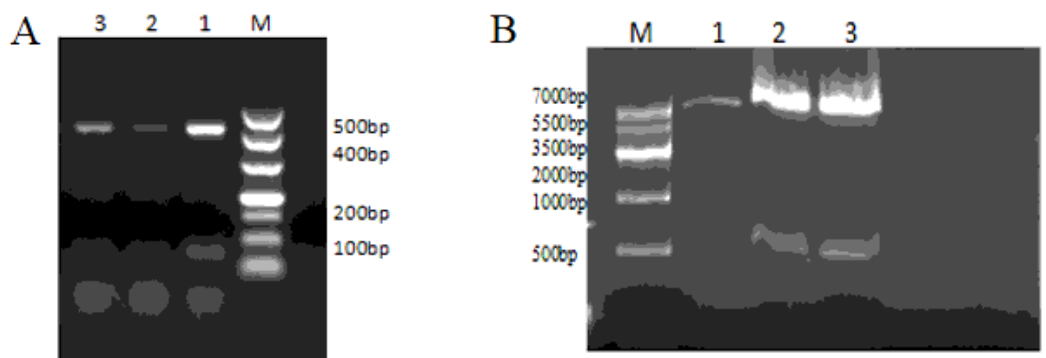

C
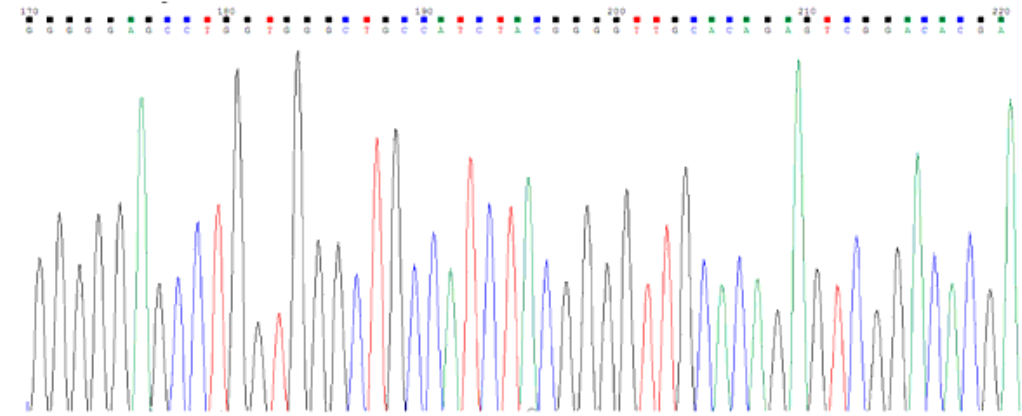

$\mathrm{D}$

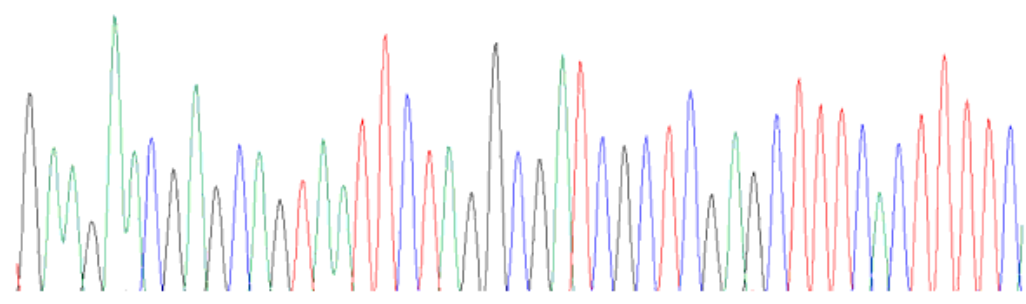

Figure 2

PCR analysis of recombinant bacteria culture and identification of psiCHECK囚-2 Vector double digests and sequencing. (A) PCR analysis of recombinant bacteria culture. Note: Markers are labeled as M (DL500), lanes 1, 2 , and 3 are TRAF6 genes. (B) Agarose gel electrophoresis of psiCHECK囚-2 Vector double digests. Note: M: Marker IV. Lane 1: original plasmid; lane 2: mutant plasmid digestion; 3: Wild-type plasmid digestion. (C) Electropherogram of the Luciferase reporter vector psiCHECK囚-2-TRAF6 sequencing. (D) Electropherogram of the Luciferase reporter vector psiCHECK》-2-TRAF6-mut sequencing 


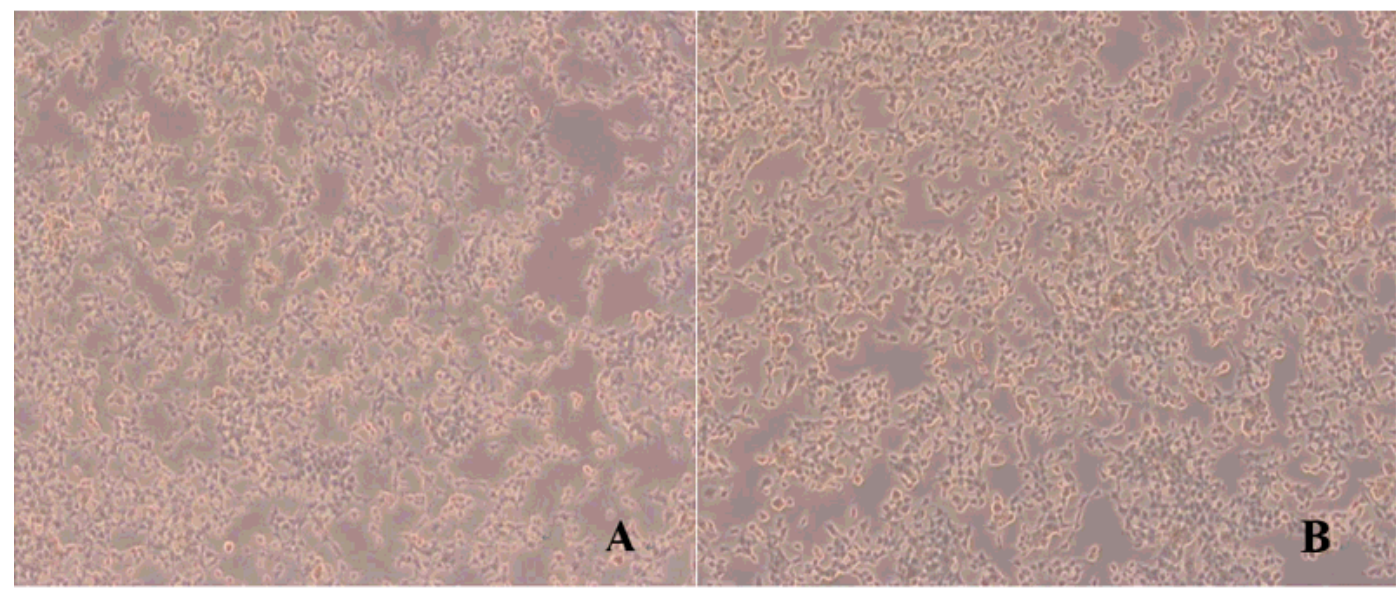

\section{Figure 3}

Images of $293 \mathrm{~T}$ cells before and after transfection (10x). (A) $2 \mathrm{~h}$ before transfection. (B) $6 \mathrm{~h}$ after transfection

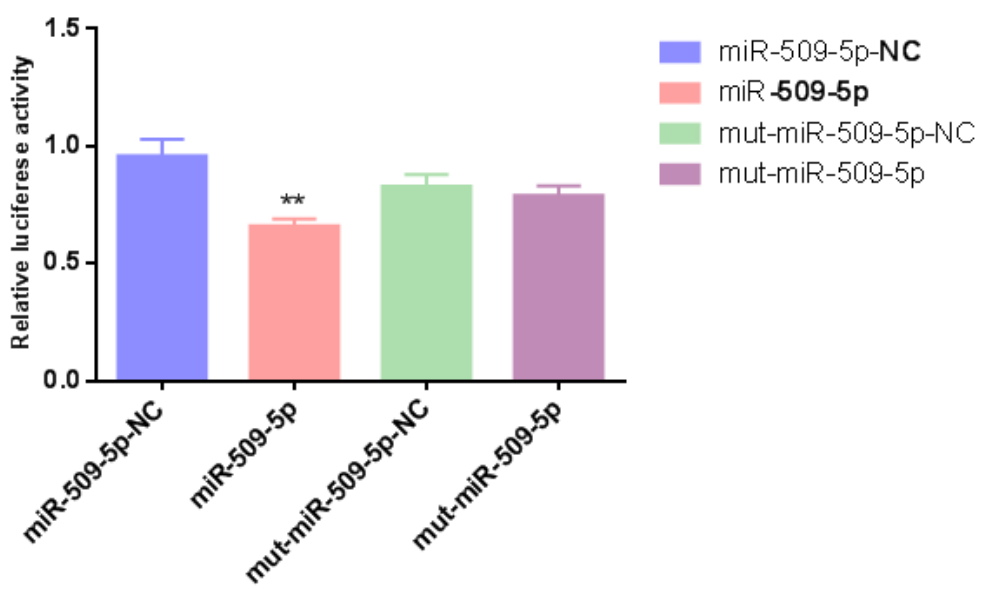

\section{Figure 4}

Double Luciferase activity was analyzed of miR-509-5p target gene

A

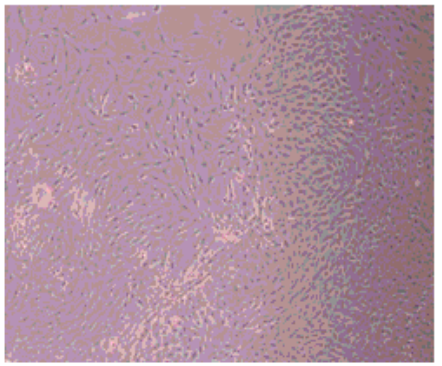

$\mathrm{B}$

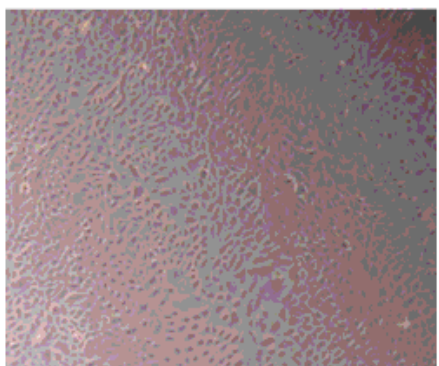

C

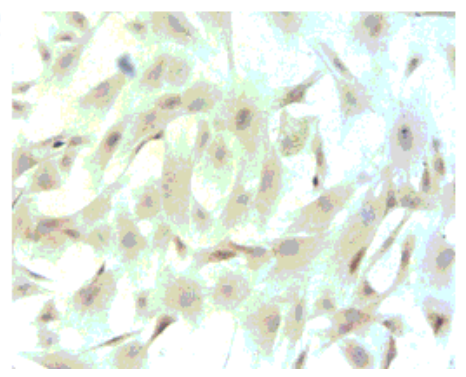

\section{Figure 5}

Primary culture and immunohistochemical staining of sheep respiratory tract epithelial cells. (A) Primary culture of sheep respiratory mucosal epithelial cells (10x). (B) Sheep respiratory epithelial cells spread over the bottom of the culture dish (10x). (C) Immunohistochemical staining of sheep respiratory epithelial cells to recognize keratin 11 (40x) 

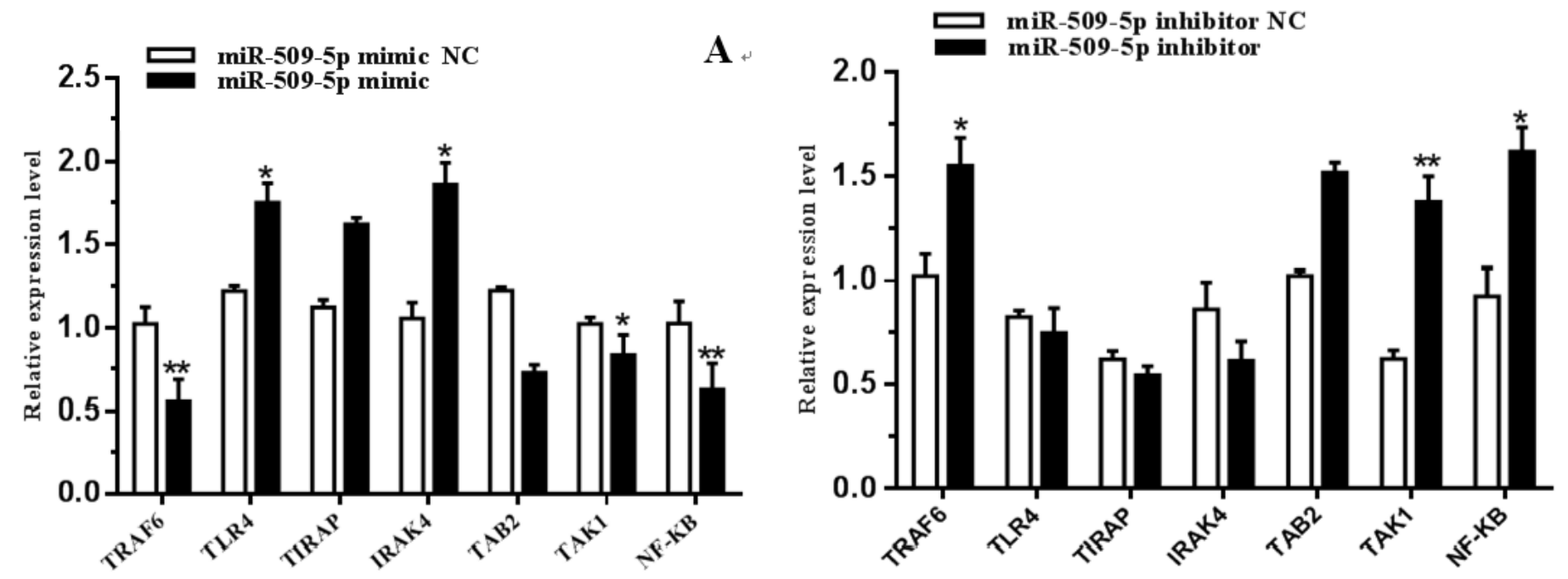

Figure 6

MicroRNA (miRNA) miR-509-5p affects NF-KB signaling pathway gene mRNA expression. (A) Effect of the miR-509-5p mimic and its negative control (NC) on NF-KB signaling pathway gene mRNA expression. (B) Effect of the miR-509-5p inhibitor and its NC control on NF-KB signaling pathway gene mRNA expression "BB" type.txt TGRGACTATCCGGARGCCTGCCQTGTGATTCCTGTGGI 116

Fj97762.txt [GagaCTATCCGGaAgaCCTGCCOGGIGaTIGCCTGTGG] 120

Consensus rgagactatccggaagacctgcce gtgattgcctgtggt

Figure 7

Results of the comparison of PCR product sequencing 

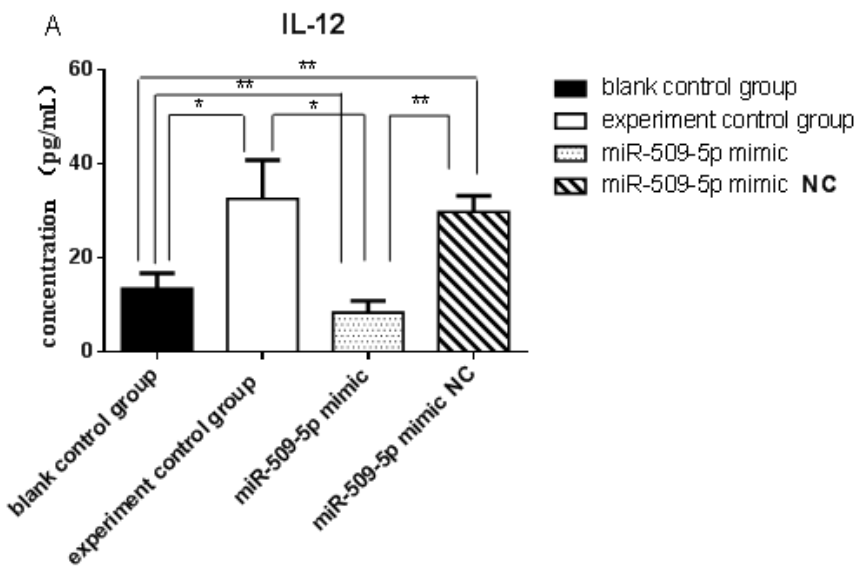

$\mathrm{B}$
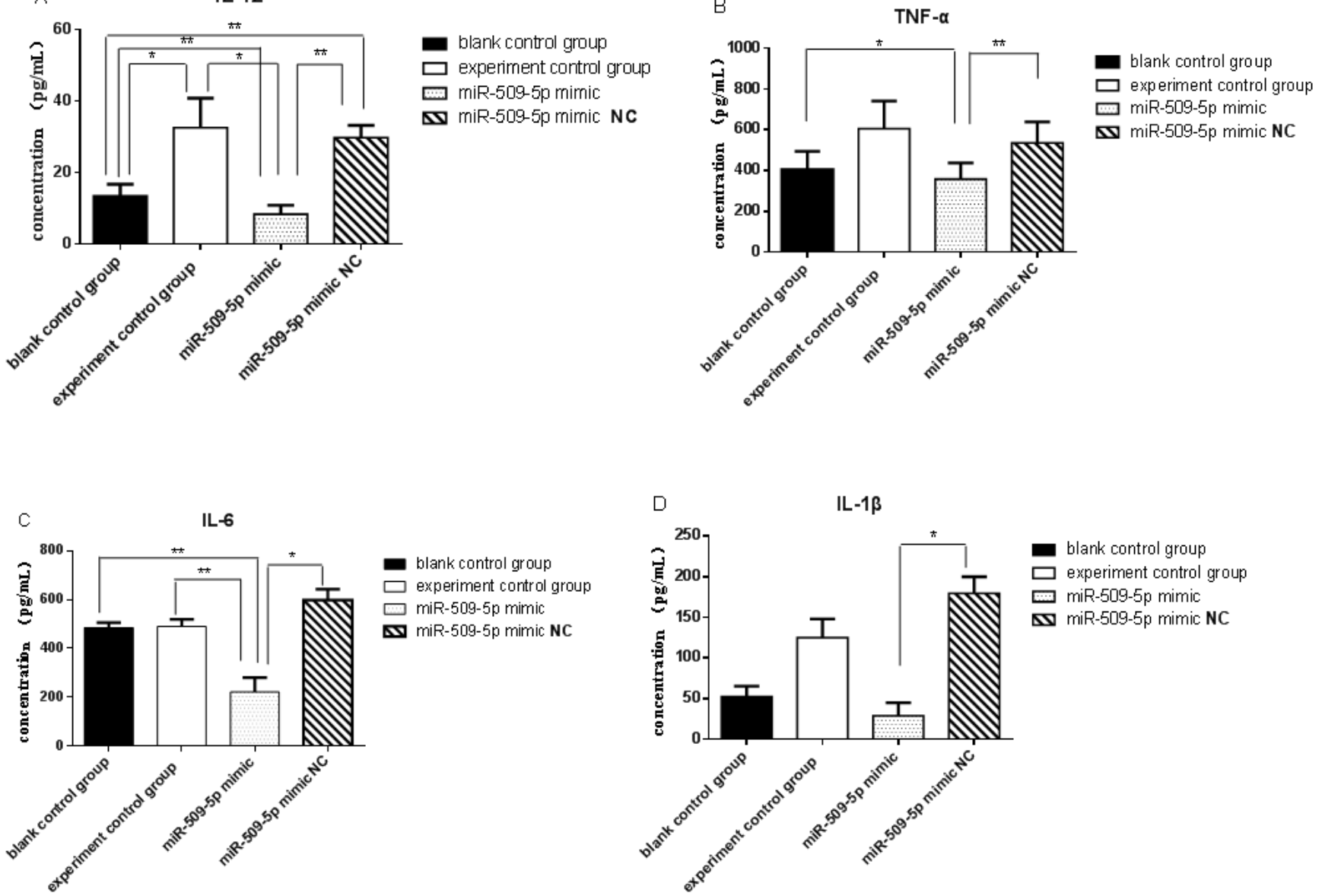

Figure 8

Changes of immune factor expression in cell culture supernatant. (A) The changes in IL-12 (pg/mL) in cell culture supernatants. (B) The changes in TNF- $\alpha$ $(\mathrm{pg} / \mathrm{mL})$ in cell culture supernatants. (C) The changes in $\mathrm{IL}-6(\mathrm{pg} / \mathrm{mL})$ in cell culture supernatants. (D) The changes in IL-1 $(\mathrm{pg} / \mathrm{mL})$ in cell culture supernatants
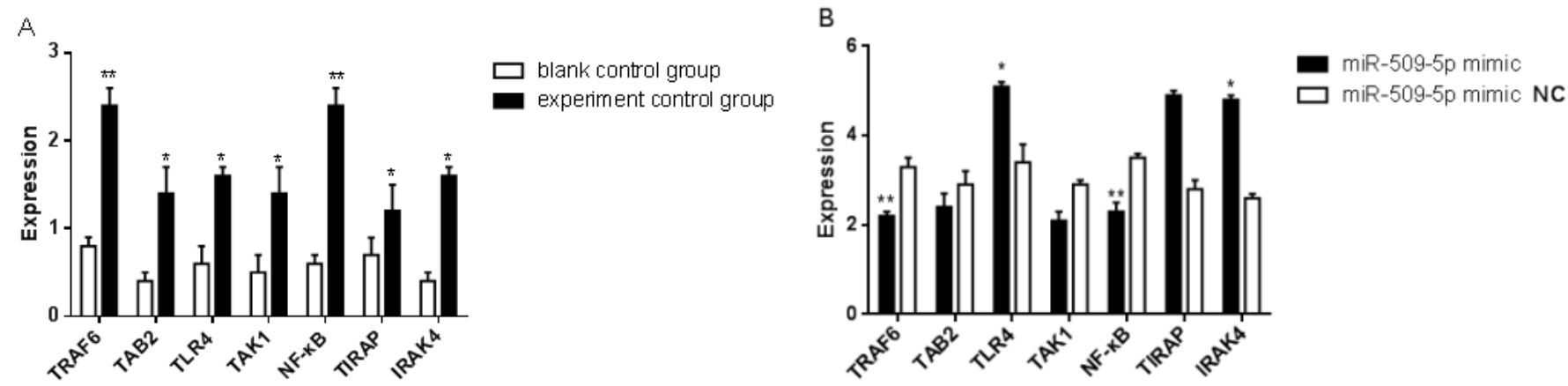

Figure 9

Changes in the mRNA expression of TRAF6 and other genes in the NF-KB pathway. (A) Detection of the expression of NF-KB pathway-related signal molecule in respiratory mucosal epithelial cells induced by Mycoplasma ovipneumoniae (MO) at the mRNA level. (B) Effect of miR-509-5p on the expression of NF-KB pathway genes at mRNA level. 


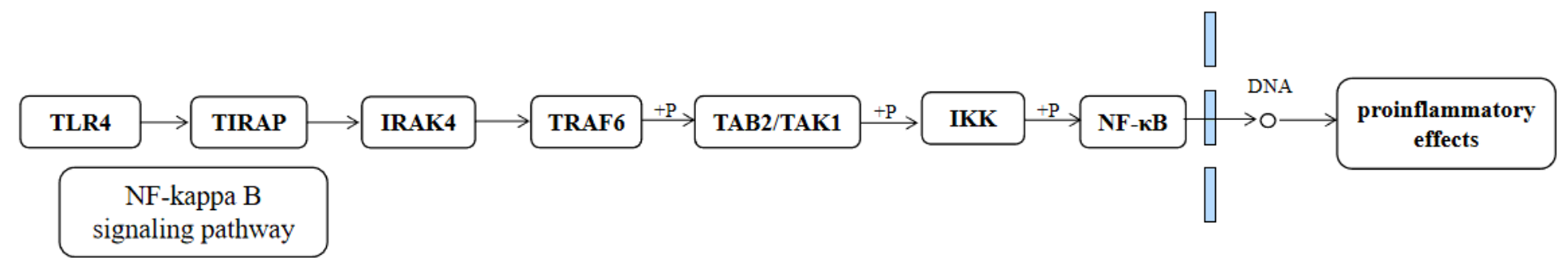

\section{Figure 10}

Schematic diagram of the NF-KB signaling pathway

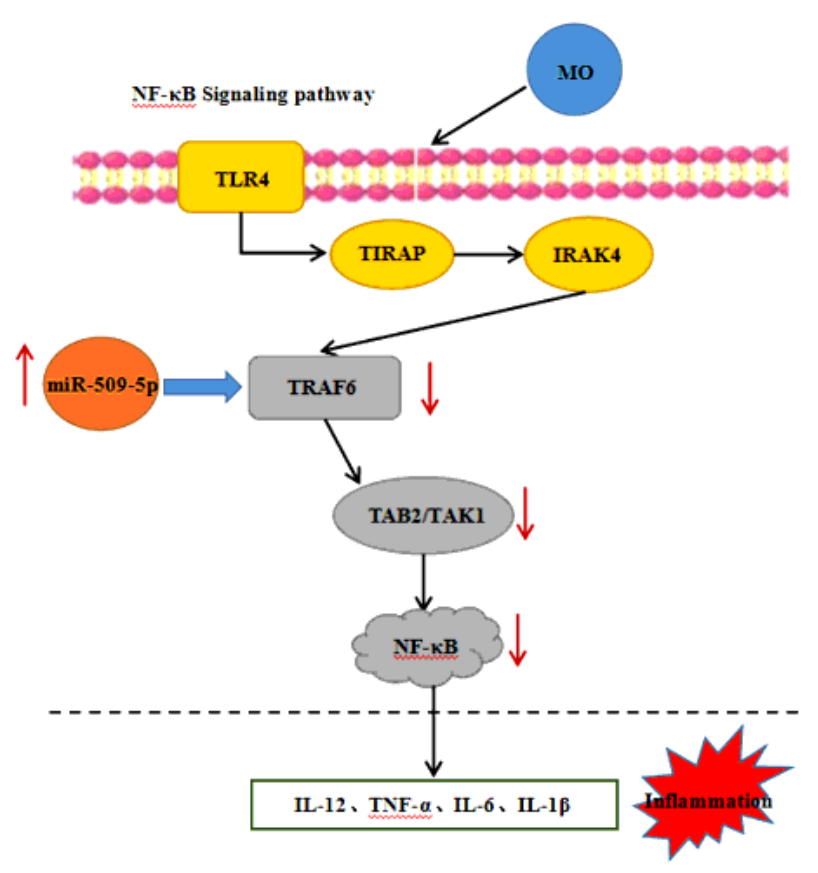

Figure 11

Schematic diagram showing how miR-509-5p targets TRAF6 to resist Mycoplasma ovipneumoniae (MO) infection in in sheep 